\title{
ON THE ARENS PRODUCT AND COMMUTATIVE BANACH ALGEBRAS
}

\author{
PAK-KEN WONG
}

\begin{abstract}
The purpose of this note is to generalize two recent results by the author for commutative Banach algebras. Let $A$ be a commutative Banach algebra with carrier space $X_{A}$ and $\pi$ the canonical embedding of $A$ into its second conjugate space $A^{* *}$ (with the Arens product). We show that if $A$ is a semisimple annihilator algebra, then $\pi(A)$ is a two-sided ideal of $A^{* *}$. We also obtain that if $A$ is a dense two-sided ideal of $C_{0}\left(X_{A}\right)$, then $\pi(A)$ is a two-sided ideal of $A^{* *}$ if and only if $A$ is a modular annihilator algebra.
\end{abstract}

1. Notation and preliminaries. Notation and definition not explicitly given are taken from Rickart's book [5].

For any subset $E$ of a Banach algebra $A$, let $L_{A}(E)$ and $R_{A}(E)$ denote the left and right annihilators of $E$ in $A$, respectively. Then $A$ is called a modular annihilator algebra if, for every maximal modular left ideal $I$ and for every maximal modular right ideal $J$, we have $R_{A}(I)=(0)$ if and only if $I=A$ and $L_{A}(J)=(0)$ if and only if $J=A$ (see [2, p. 568, Definition]).

Let $A$ be a Banach algebra, $A^{*}$ and $A^{* *}$ the conjugate and second conjugate spaces of $A$, respectively. The Arens product on $A^{* *}$ is defined in stages according to the following rules (see [1]). Let $x, y \in A, f \in A^{*}, F$, $G \in A^{* *}$.

(a) Define $f \circ x$ by $(f \circ x)(y)=f(x y)$. Then $f \circ x \in A^{*}$.

(b) Define $G \circ f$ by $(G \circ f)(x)=G(f \circ x)$. Then $G \circ f \in A^{*}$.

(c) Define $F \circ G$ by $(F \circ G)(f)=F(G \circ f)$. Then $F \circ G \in A^{* *}$.

$A^{* *}$ with the Arens product $\circ$ is denoted by $\left(A^{* *}, \circ\right)$. Let $\pi$ be the canonical embedding of $A$ into $A^{* *}$. Then $\pi(A)$ is a subalgebra of $\left(A^{* *}, \circ\right)$.

Let $A$ be a Banach algebra. For each element $x \in A$, let $\operatorname{Sp}_{A}(X)$ denote the spectrum of $x$ in $A$. If $A$ is commutative, $X_{A}$ will denote the carrier space of $A$ and $C_{0}\left(X_{A}\right)$ the algebra of all complex-valued continuous functions on $X_{A}$, which vanish at infinity; $C_{0}\left(X_{A}\right)$ is a commutative $B^{*}$-algebra.

In this paper, all algebras and linear spaces under consideration are over the complex field $C$.

Received by the editors April 17, 1972.

AMS (MOS) subject classifications (1970). Primary 46C05; Secondary 46K99.

Key words and phrases. Arens product, modular annihilator, annihilator algebra, carrier space.

(c) American Mathematical Society 1973 
2. The results. Our first result is a generalization of $[7$, p. 82 , Theorem 3.3] for commutative Banach algebras.

THEOREM 2.1. Let $A$ be a semisimple commutative annihilator Banach algebra. Then $A$ is a two-sided ideal of $\left(A^{* *}, \circ\right)$.

Proof. Let $X_{A}$ be the carrier space of $A$ and let $B=C_{0}\left(X_{A}\right)$. Since $A$ is an annihilator algebra, it is well known that $X_{A}$ is discrete and therefore $B$ is a dual $B^{*}$-algebra by $[6$, p. 532 , Theorem 4.2$]$. Let $|\cdot|$ be the norm on $B$. Then the given norm $\|\cdot\|$ majorizes $|\cdot|$ on $A$. Considering $A$ as a subalgebra of $B$, we show that $A$ is dense in $B$. Let $x \neq 0$ be a hermitian element in $B$. Then it is known that $\mathrm{Sp}_{B}(x)$ has no nonzero limit points and so it is a countable set (see [8, p. 826, Theorem 3.1]). Therefore it follows from [5, p. 111, Theorem (3.1.6)] that $\left\{\alpha(x): \alpha \in X_{A}\right\}$ is countable. Denote those $\alpha \in X_{A}$ for which $\alpha(x) \neq 0$ by $\alpha_{1}, \alpha_{2}, \cdots$. Then $\alpha_{n}(x) \rightarrow 0$ as $n \rightarrow \infty$. For each $\alpha_{n}$, by Šlov's theorem [5, p. 168, Theorem (3.6.3)], there exists a nonzero idempotent $e_{n}$ in $A$ such that $e_{n}\left(\alpha_{n}\right)=1$ and $e_{n}(\alpha)=0$ for all $\alpha \neq \alpha_{n}$.

Let $\varepsilon>0$ be given. Then there exists a positive integer $N$ such that $\left|\alpha_{n}(x)\right|<\varepsilon$ for all $n \geqq N$. Let $y=\sum_{n=1}^{N} \alpha_{n}(x) e_{n}$. Then $y \in A$ and it is easy to see that

$$
|x-y|=\sup \left\{|\alpha(x)-\alpha(y)|: \alpha \in X_{A}\right\}<\varepsilon .
$$

Therefore it follows now that $A$ is dense in $B$. Since $A$ is a dual $B^{*}$-algebra, by [7, p. 82, Theorem 3.3], $B$ is a two-sided ideal of $B^{* *}$ (with the Arens product). Therefore, by [7, p. 82, Lemma 3.2], $A$ is a two-sided ideal of $\left(A^{* *}, \circ\right)$ and the proof is complete.

The following result is a generalization of $[8, \mathrm{p} .830$, Theorem 5.2] for commutative Banach algebras.

THEOREM 2.2. Let $A$ be a commutative Banach algebra such that $A$ is a dense two-sided ideal of $C_{0}\left(X_{A}\right)$. Then $A$ is a two-sided ideal of $\left(A^{* *}, \circ\right)$ if and only if $A$ is a modular annihilator algebra.

Proof. Let $B=C_{0}\left(X_{A}\right)$ and let $|\cdot|$ be the norm on $B$. By $[3$, p. 3, Theorem 2.3], there exists a constant $k$ such that $\|a b\| \leqq k\|a\||b|$ and $\|a b\| \leqq k|a|\|b\|$ for all $a, b \in A$. It is easy to see that $A$ is a semisimple algebra. Suppose $A$ is a two-sided ideal of $\left(A^{* *}, \circ\right)$. Then by the proof of $\left[8\right.$, p. 829, Lemma 5.1], we can show that $X_{A}$ is discrete and therefore $A$ is a modular annihilator algebra (see [2, p. 578, Example 8.4]). Conversely suppose $A$ is a modular annihilator algebra. Then, by [2, p. 569, Theorem $4.2(6)], X_{A}$ is discrete in the hull-kernel topology and therefore $X_{A}$ is discrete in the finer Gelfand topology. Hence $B$ is a dual $B^{*}$-algebra. Now by the argument in $[8$, p. 830 , Theorem 5.2], we can show that $A$ is a twosided ideal of $\left(A^{* *}, \circ\right)$, and this completes the proof. 
COROLlaRy 2.3. Let $A$ be as in Theorem 2.2. If $A$ is reflexive and has an approximate identity, then $A$ is finite dimensional.

Proof. By [4, p. 855, Lemma 3.8], $A$ has an identity element. Also it follows from Theorem 3.2 that $A$ is a modular annihilator algebra and therefore it is finite dimensional by [2, p. 573, Proposition 6.3].

Let $G$ be a compact abelian group with the Haar measure and let $A=$ $L_{2}(G)$. Then it is well known that $A$ is reflexive and $A$ is a dense two-sided ideal of $C_{0}\left(X_{A}\right)$. Also if $A$ is infinite dimensional, $A$ has no approximate identity.

\section{REFERENCES}

1. R. F. Arens, The adjoint of a bilinear operation, Proc. Amer. Math. Soc. 2 (1951), 839-848. MR 13, 659.

2. B. A. Barnes, Modular annihilator algebras, Canad. J. Math. 18 (1966), 566-578, MR 33 \#2681.

3. - Banach algebras which are ideals in a Banach algebra, Pacific J. Math. 38 (1971), 1-7.

4. P. Civin and B. Yood, The second conjugate space of a Banach algebra as an algebra, Pacific J. Math. 11 (1961), 847-870. MR 26 \#622.

5. C. E. Rickart, General theory of Banach algebras, University Series in Higher Math., Van Nostrand, Princeton, N.J., 1960. MR 22 \#5903.

6. B. J. Tomiuk and P. K. Wong, The Arens product and duality in $B^{*}$-algebras, Proc. Amer. Math. Soc. 25 (1970), 529-535. MR 41 \#4256.

7. P. K. Wong, On the Arens product and annihilator algebras, Proc. Amer. Math. Soc. 30 (1971), 79-83.

8. - Modular annihilator $A^{*}$-algebras, Pacific J. Math. 37 (1971), 825-834.

Department of Mathematics, McMaster University, Hamilton, Ontario, CANADA

Current address: Department of Mathematics, Seton Hall University, South Orange, New Jersey 07079 